A direct comparison of wrist actigraphy and sleep diaries in measuring sleep in healthy adults.

\title{
Zainab Alyobi
}

$\&$

Susan Mary Sherman

School of Psychology, Keele University, Keele, Staffordshire, ST5 5BG, UK.

Correspondence to Susan M Sherman: s.m.sherman@keele.ac.uk 


\begin{abstract}
Measuring sleep and sleep quality is an important diagnostic and monitoring tool, and a number of different methods for measurement have been developed over the last half-century. Two prevalent methods include wrist actigraphy and sleep diaries. Both methods can be applied in different circumstances, but both have strengths and weaknesses. This study aimed to identify the extent to which there is congruence in the scores achieved by each method of sleep measurement. Sixty-eight respondents were asked to wear a wrist actigraphy and complete a sleep diary over the course of five days. There was a significant difference between the mean scores achieved using each measurement method, with actigraphy scores indicating lower total sleep time (TST) than diaries. However, this difference was not consistently present when the scores were compared on a day by day basis. Participant adherence is likely to fluctuate over the course of a sleep study and may undermine the accuracy of sleep diaries.
\end{abstract}

\title{
Keywords
}

sleep, actigraphy watch, self-report, sleep duration 


\section{Introduction}

Whilst Polysomnography (PSG) constitutes the gold standard in detecting sleep and related interruptions, there are limitations to its applications. Since it is often deployed within sleep labs, it is unsuitable for long term or longitudinal studies, it may also introduce elements of bias since it does not represent the monitoring of sleep within a natural setting. As such, there is a need for alternative methods of sleep monitoring which do not compromise on accuracy, but which are cost effective and relatively straightforward to implement. Two commonly deployed methods are actigraphy and self-reported sleep diaries. Actigraphy refers to the use of a piezoelectric accelerometer which detects the presence or absence of movement (Sadeh, 2011). The ability to wear a suitable device on the wrist means that "actigraphy can conveniently record continuously for 24 hours a day, for days, weeks or even longer" (Ancoli-Israel, 2003, p. 342). Whilst in the early 1970s - when actigraphy was first introduced - there were concerns that actigraphy was plagued with methodological issues, increasing updates to the literature (and improvements in the technology) make it one of the most reliable means of measuring sleep (Ancoli-Israel, 2003).

There are a variety of elements of sleep that actigraphy seeks to measure; total sleep time (TST), sleep latency (SL - the time taken to fall asleep), and wake after sleep onset (WASO - the extent to which sleep is disturbed after initially falling asleep). A number of studies have been conducted in order to understand what levels of reliability and validity are achieved by the technology. For instance, Fonseca et al. (2017) identified that when compared to PSG, actigraphy watches tended to overestimate TST and underestimate WASO. However, other studies, such as that conducted by Vallieres and Morin (2003) have drawn different conclusions, specifically that total wake time was overestimated, and TST was underestimated. The latter study drew its sample from a population already suffering from sleep disturbances, which suggests that actigraphy's suitability might be dependent on the nature of the condition which it is seeking to address.

In terms of sleep measurement, there are not just questions of how effective actigraphy is in-and-of-itself, but how well it compares to alternative measurement approaches. It has been identified that PSG is the 'gold standard' within the field, but 
sleep diaries are a commonly used alternative (Peltz, Rogge \& O'Connor, 2018). These constitute a 'self-report' measure, in which the subject reports their previous night's sleep in a record.

In relation to a comparison of the two approaches - which is the central goal of this study - there have been a number of studies which have sought to engage in a comparative analysis in a number of different contexts. There are conflicting results amongst these studies. Maich, Lachowski and Carney (2018) found that there was a tendency for actigraphy to give measures of TST that were shorter than that recorded by diary. A similar finding was also found by Short, Gradisar, Lack, Wright and Carskadon (2012) and Campanini et al. (2017), however, studies byKawada (2008) and Vallieres and Morin (2003) found the opposite.

Most recently, Thurman et al (2018) carried out an assessment of 30 healthy adults over a 16-week period using wrist actigraphy and sleep diary. They also found that the wrist actigraphy showed a shorter TST than sleep diary. Another issue they identified was that compliance with the sleep diary became less consistent over time. At the start of the study, about $87 \%$ of subjects completed their sleep diary, and by the end of the study the percentage dropped to $66 \%$. The results of this study presented the difference between the actigraphy and sleep log measurements for the whole sampling period (4 months). Investigating the agreements in both methods for on a daily or weekly basis might provide a more fine-grained understanding of the extent to which subjective and objective sleep measurements tend to agree in measuring sleep variables.

Actigraphy and sleep diaries hold strong promise for the measurement of sleep and wakefulness, but with caveats. Most of the studies to date has been inclined to engage in a comparison of actigraphy with sleep diaries by evaluating the overall TST measurements (i.e. for the whole days) derived from the actigraphy and sleep diaries. This is likely to limit our understanding of how much concordance there is between the scores generated by each tool on a day by day basis. There is a need for additional investigation to identify the comparative value of each approach. The current study 
aimed to identify in more detail, the extent to which there is congruence in TST scores between actigraphy and sleep diary in healthy young adults for five consecutive days.

\section{Method}

The current data were collected as a part of a bigger study investigating the effect of sleep on false memory.

\section{Participants}

68 native English-speaking students and staff from Keele university were recruited to participate in the main study. The age of the participants ranged from 18-65 years $(M=20, S D=6.26)$ and there were 52 females. Participants received either 1 hour of course credit or $£ 10$ for their participation. The study was approved by the Keele University Ethics Review Panel.

\section{Measures}

An actigraphy watch and sleep diary were used for five days to collect data on TST. Sleep duration was measured by Motion Watch8 (CamNtech Ltd, UK). Participants were asked to wear the actigraphy watch on their non-dominant hand, to keep it on all the time until the end of the study and not to switch the watch between hands. They were also asked to press the marker button (used to indicate the specific time) when they went to bed at night and when they woke up in the morning. The actigraphy output was automatically scored using the manufacturer's software (MotionWare 1.2.5) with 30 seconds of epoch length.

Participants also completed the National Sleep Foundation sleep diary upon awaking, which asked about nightly bedtime and wake time. The diary was used to collect key information on sleep duration, timings of going to bed and waking up in order to compare the watch findings with participant-reported sleep patterns.

\section{Procedure}

Participants who signed up for the larger study on false memory, were invited to the lab, where they were given detailed information about the study and instructions on how to wear the watch and complete the sleep diary. They then left and wore the 
actigraphy watch and completed the sleep diary for five consecutive days before returning to the lab for the first part of the false memory study.

\section{Results}

The means and standard deviations for the TST are presented in Table 1. A related ttest revealed a significant difference in the overall TST between the actigraphy watch $490 \pm 54 \mathrm{~min}$ and sleep diary $507 \pm 58 \mathrm{~min}(\mathrm{t}(67),-3.15, \mathrm{p}<0.01)$.

\begin{tabular}{c|cc}
\multicolumn{2}{c}{ M \pm SD (N=68) } \\
\hline DAY 1 & Actigraphy & Sleep Diary \\
DAY 2 & $488 \pm 117$ & $497 \pm 118$ \\
DAY 3 & $475 \pm 102$ & $496 \pm 91$ \\
DAY 4 & $515 \pm 96$ & $552 \pm 112$ \\
DAY 5 & $496 \pm 100$ & $512 \pm 112$ \\
OVERALL & $456 \pm 89$ & $485 \pm 110$ \\
\hline
\end{tabular}

Table 1. The means and standard deviations of total sleep time (TST) in minutes for actigraphy watch and sleep diary

However, related t-tests run on the daily scores reveals that the average difference between actigraphy and sleep diaries does not remain consistently different (see Figure1). There was no significant difference between actigraphy and diary for TST on day one ( $\mathrm{t}(67),-.85, p=0.394)$, day two $(\mathrm{t}(67),-1.84, p=0.069)$ and day four $(\mathrm{t}(67)$, $1.38, p=0.172)$. There was a significant difference on day three $(\mathrm{t}(67),-2.91, p<0.01)$ and day five ( $\mathrm{t}(67),-2.95, p<0.01)$ with the diary recording a greater mean TST than actigraphy, with mean reported differences of $37 \pm 105 \mathrm{~min}$ and $28 \pm 79 \mathrm{~min}$ respectively. 


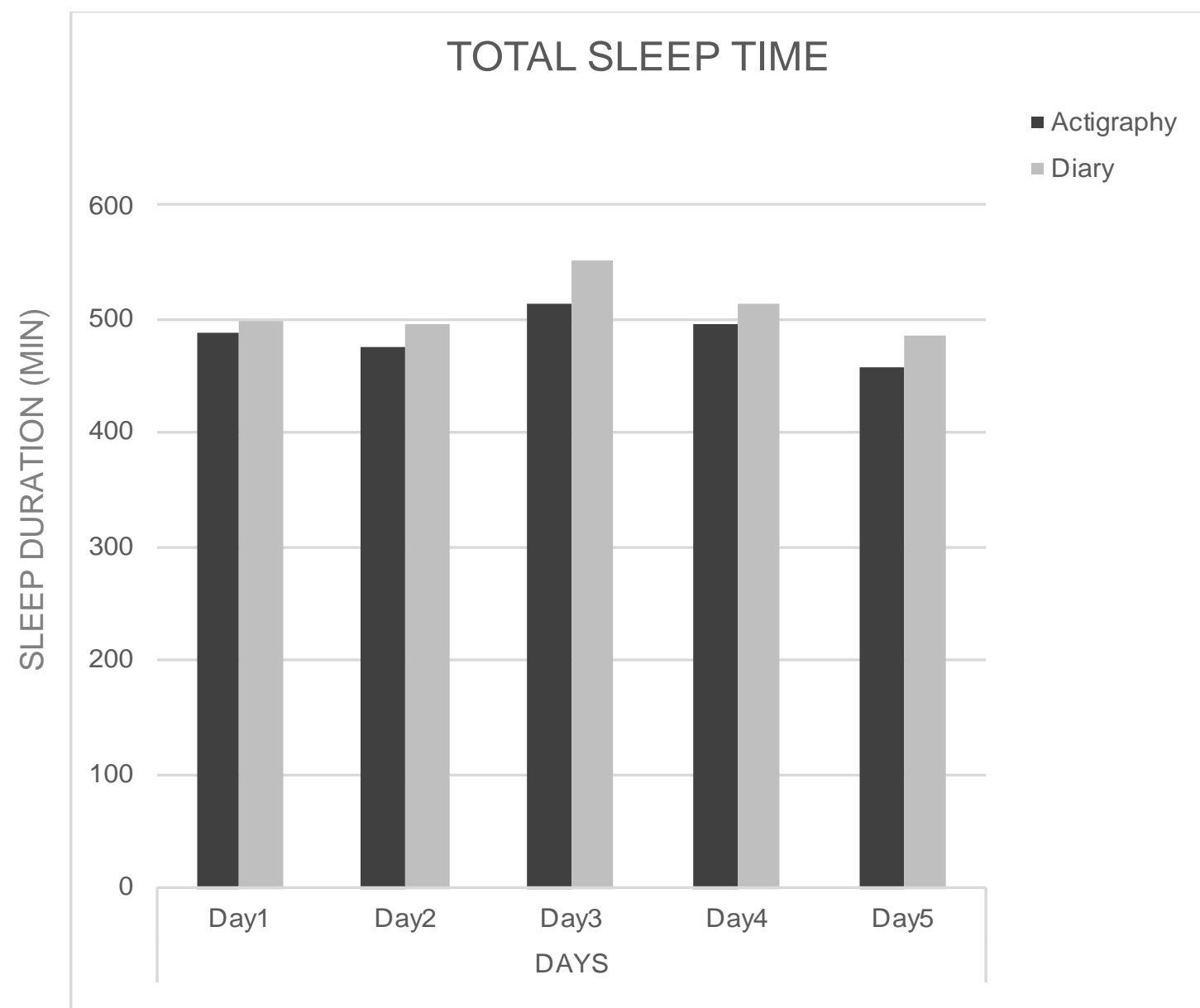

Figure 1. TST mean across the five days as assessed via Actigraphy and Sleep Diary.

The relationship between actigraphy and sleep diary was further explored with Pearson correlation coefficients. Results demonstrated a strong positive correlation 
between the TST reported by both methods for overall TST $(r=0.69, p<0.01)$, day one TST $(r=0.68, p<0.01)$ and day five TST $(r=0.70, p<0.01)$; and a moderate positive correlation between the TST scores recorded on day two $(r=0.53, p<0.01)$, day three $(r=0.50, p<0.01)$ and day four, $(r=0.57, p<0.01)$.

\section{Discussion}

This current study measured the TST derived from actigraphy watches and sleep diaries for five consecutive days from 68 healthy individuals. The findings of this study are consistent with those found by Short et al. (2012), Campanini et al. (2017) and Maich, Lachowski \& Carney (2018) that is actigraphy generally measured a lower overall TST relative to that recorded by sleep diary. It should be noted from the results that although there are differences between the actigraphy and diary reporting scores generated, without reference to a third 'gold standard' level of reporting (i.e. PSG), it is not possible to say which of these levels was the most accurate. Thus, one cannot state that actigraphy 'underestimated' or that diary reporting 'overestimated'.

Interestingly, the differences between actigraphy scores and diary scores are not consistently different. Across the five days, we found that there was no difference in TST scores between the actigraphy and diary on the first, second and fourth days. While a significant difference was observed on the third and fifth days. This suggests that there is not a consistent level of differentiation between the scores generated from actigraphy and sleep diaries, but instead variation on a day by day basis.

There are a number of factors which may contribute to the unevenness of these scores. The first of these is that there may be an element of 'getting used to' the idea of assessing sleep patterns - with a greater awareness of sleep being developed as the study develops. However, the fact that the diary results showed a differentiation from actigraphy measures on both third and fifth days precludes this assessment from the data given. An alternative explanation given by Lawrence and Muza (2018) is that respondents may simply fail to complete their diaries, and only do so on the final day before they are due to submit results. They state that this can lead "to inaccuracies due to problems with recall and a tendency to just put rough guesses to complete the document" (p.180). Interpreting our results in light of this suggestion, it is possible that 
the participants are more diligent at the start of the study, completing the sleep diary daily for the first couple of days, but then becoming less diligent, having to complete it retrospectively. If this is the case, caution is needed when using sleep diaries as participants may become less accurate in their record-keeping over time. Another possible explanation is that the present of actigraphy might have an impact on participants' performance on the diary. In the first days of the study, they may feel very conscious that their sleep is being monitored, as a result they are more compliant in recording it, but over time that feeling fades. If this explanation is correct, then caution is needed relying solely on self-reported TST.

Despite these inconsistencies, we found strong to moderate correlations in TST between the actigraphy and the sleep diary both overall and across each of the five days. Taken together, the results of our study suggest that the broad pattern of sleep is accurately recorded, but that the precise details are not recorded effectively and that the level of precision will fluctuate across even a 5-day recording span. If we assume that the objective actiwatch is the more consistent of the two recording methods, our findings suggest that the biggest problem with sleep diaries is not an inherent difficulty in the subjective assessment of sleep, but instead the fact that non-compliance and/or misrepresented data might undermine conclusions about the sleep patterns of respondents, especially where these conclusions rest on details such as TST. A modification to this method might be to send participants a prompt every morning throughout the diary recording period to record their sleep, possibly electronically.

An evaluation of the day to day fluctuations in consistency between TST recorded by actigraphy and sleep diaries over 5 days suggests that these fluctuations may increase over time which may have implications for the accuracy of longitudinal studies using sleep diaries. Further studies are required to confirm these findings and explore ways to overcome any fluctuations.

\section{Acknowledgements}

We thank the Saudi government, represented by the Ministry of Higher Education and Jeddah University for the financial support. We would also like to thank the 
Psychology department, Keele University, for their cooperation during the data collection. 


\section{References}

Ancoli-Israel, S., Cole, R., Alessi, C., Chambers, M., Moorcroft, W., \& Pollak, C. P. (2003). The role of actigraphy in the study of sleep and circadian rhythms. Sleep, 26(3), 342-392.

Campanini, M. Z., Lopez-Garcia, E., Rodríguez-Artalejo, F., González, A. D., Andrade, S. M., \& Mesas, A. E. (2017). Agreement between sleep diary and actigraphy in a highly educated Brazilian population. Sleep Medicine, 35, 2734.

Fonseca, P., Weysen, T., Goelema, M. S., Møst, E. I., Radha, M., Lunsingh Scheurleer, C., ... \& Aarts, R. M. (2017). Validation of photoplethysmographybased sleep staging compared with polysomnography in healthy middle-aged adults. Sleep, 40(7), zsx097.

Kawada, T. (2008). Agreement rates for sleep/wake judgments obtained via accelerometer and sleep diary: a comparison. Behavior Research Methods, 40(4), 1026-1029.

Lawrence, G., \& Muza, R. (2018). Assessing the sleeping habits of patients in a sleep disorder centre: a review of sleep diary accuracy. Journal of Thoracic Disease, 10(Suppl 1), S177.

Maich, K. H., Lachowski, A. M., \& Carney, C. E. (2018). Psychometric properties of the consensus sleep diary in those with Insomnia disorder. Behavioral Sleep Medicine, 16(2), 117-134.

Peltz, J. S., Rogge, R. D., \& O'Connor, T. G. (2018). Adolescent sleep quality mediates family chaos and adolescent mental health: A daily diary-based study. Journal of Family Psychology.

Sadeh, A. (2011). The role and validity of actigraphy in sleep medicine: an update. Sleep Medicine Reviews, 15(4), 259-267.

Short, M. A., Gradisar, M., Lack, L. C., Wright, H., \& Carskadon, M. A. (2012). The discrepancy between actigraphic and sleep diary measures of sleep in adolescents. Sleep Medicine, 13(4), 378-384.

Thurman, S. M., Wasylyshyn, N., Roy, H., Lieberman, G., Garcia, J. O., Asturias, A., ... \& Mednick, S. C. (2018). Individual differences in compliance and agreement for sleep logs and wrist actigraphy: A longitudinal study of naturalistic sleep in healthy adults. PloS One, 13(1), e0191883. 
Vallières, A., \& Morin, C. M. (2003). Actigraphy in the assessment of insomnia. Sleep, 26 (7), 902-906. 